\title{
Influencing Factors of Travel Route Choice in Advanced Transportation Information Service System Using Mixed Logit Model
}

\author{
Gengyong Cao ${ }^{1}$ Chunqin Zhang ${ }^{2}$ \\ Zhejiang scientific research institute of transport, Hangzhou, 310006, china \\ School of Civil Engineering and Architecture, Zhejiang Sci-Tech University, Hangzhou, 310018, \\ China
}

\begin{abstract}
Keywords: Advanced Transportation Information Service system; traffic information engineering; travel route choice; Stated Preference survey; mixed logit model

Abstract: In order to understand how traffic information influences travel route choice. State Preference survey is carried out to investigate travelers' behavior data. Mixed logit models are established to analyze the factors influencing travel route choice, one for overall object model and the other for typical object model. The models are then verified using survey data from different range. The results confirm that travelers' socio-economic characteristics, the severity of traffic incident or congestion, travel time saved by the alternative route, the delay on the original route, road network knowledge, and the travelers' evaluation and attitude on traffic information are the main factors influencing travel route choice. There are some different influencing factors between overall object model and typical object model. The study underscores the significance of traffic information and the potential effect of traffic information on route choice, and provides the basis for traffic information dissemination methods and strategies.
\end{abstract}

\section{Introduction}

The growing traffic congestion has led to economic inefficiency, social disruption, excessive energy consumption and increased levels of pollution. To alleviate serious traffic congestion, the real-time traffic information is provided to help travelers choose the best travel route and guide them to effectively use of the existing network more efficiently. It is a feasible strategy for travel time saving and traffic congestion alleviation.

There have been many efforts over the years to obtain a more detailed understanding of how travelers decide which routes to consider and then select one to follow. Many of these have been directed towards understanding the decision mechanism that underlies travelers' route choice behavior, and establishing an appropriate model theory and model form.

One of the basic approaches to understand travelers' route choice behavior is descriptive data analysis. Data collected in the field and from driver surveys are used to infer travelers' route choice criteria and their relative importance travelers' decision-making processes. Descriptive statistics of the data form the basis of this approach ${ }^{[1 \sim 3]}$.

Another distinct approach to understand travelers' route choice behavior is to use different statistical techniques like principal component factor analysis, canonical correlations, multiple regressions and grouping techniques ${ }^{[1,4]}$.

Route choice can also be modeled as a continuous variable in a variety of ways ${ }^{[5]}$. Besides, disaggregate choice analysis methods based on random utility models have been widely applied to model travelers' route choice behavior. In this class of models, simple two and multinomial logit models are the simplest and perhaps most commonly used ${ }^{[6 \sim 11]}$. However, the IIA (independence from irrelevant alternatives) property of the simple logit model restricts its applicability to general route choice analysis. This property results from the logit model assumption that route utilities include a random error term, and that the error terms of different route are statistically independent of each other. Particularly in urban road networks, where alternative routes may overlap over significant portions of their length, the IIA property can be violated because of correlations in 
unobserved route attributes.

The mixed logit model is a highly flexible model that can approximate any random utility model $^{[12]}$. The advantage of the mixed logit model is that, by construction, it avoids the IIA property of the standard logit model. Estimation of mixed logit models is slightly more complex than that of simple logit models; software is readily available for this purpose. Therefore, the first objective of this study is to formulate a general framework for modeling travelers' route choice behavior. Toward this end, the mixed logit model is proposed to analyze the factors influencing route choice behavior, which retains the flexibility of the simple logit model, while exploiting to some extent the computational tractability of the simple logit model.

The second objective of this study is to analyze the factors that influence travelers' route choice behavior. Route choice behavior has been widely investigated both in the presence and absence of traffic information and is found to vary with: drivers' socio-economic characteristics and prior experience, availability and perception of alternate routes, travel time, traffic delays of original routes, the severity of traffic incidents, and traffic information availability ${ }^{[13 \sim 18]}$. Many of these studies have focused on route choice behavior based on stated-preference surveys, which of the survey area are specific and stationary. The city type and the regional type of the travelers may influence their route choice behavior, and will lead to the main factors influencing travel route choice, which are different with the different city type and the different regional type. However, in the existing literatures, there are almost no scholars who study this problem. So, this paper will focus on whether the city type and the regional type are the main factors influencing travel route choice.

The paper is organized in the following manner. Section 2 presents a brief description of the route choice survey conducted to collect the data used in the analyses presented in this paper. Then the preliminary analysis of the data has been carried out. Section 3 proposes mixed logit model to model travel route choice. In section 4, the modeling results are discussed. Section 5 presents some concluding comments and opportunities for future research.

\section{Survey Design}

Several options were considered for collecting data. The use of an interactive travel simulator was discounted because, although its results might have been superior in some aspects. An advantage of using stated intention questionnaires instead of a route-choice simulator was that the questions were designed to relate to the travelers' actual journey, rather than a "standard" journey.

An Stated Preference (SP) experiment offers decision-makers a series of hypothetical scenarios to be evaluated, usually in the form of discrete choices between travel alternatives, and the alternatives are characterized by variables whose effects on travel behavior we wish to examine. SP techniques have been extensively applied to the analysis of mode choice ${ }^{[19]}$ but with a significant number of applications to motorists' route choices ${ }^{[20 ~ 22]}$. A Revealed Preference (RP) survey based on motorists' actual choices would not have been a practical pro-position because, even if we had been able to persuade an operator to display our desired range of messages, we could not have controlled the external factors nor afforded the interview costs. Thus, this study has made use of the SP techniques in order to evaluate drivers' route choice responses to information on road traffic conditions.

\section{Data Collection}

Data to analyze the requirements from the user's point of view were collected in a two-stage survey. Before the formal investigation, a small-scale pilot survey was organized firstly in order to ensure the quality. Then, according to the respondents' feedback, the questionnaire and investigation scheme have been adjusted. In the second phase, face-to-face interviews were conducted in two parts. Each questionnaire asked for the following information:

1) Traveler socio-economic characteristics

They include: age, gender, marital status, education level, occupation, household incomes, vehicle type, city type and regional type. 
The division standard of the city type is based on the size of the population. The urban resident population of the small cities is 50 million or less, 50 million to 100 million for the medium-sized cities, 100 million to 300 million for the big cities, 300 million to 1000 million for the megalopolis. The division standard of the regional type is based on the administrative region, and the regional type is divided into seven categories, which include: Northeast、North china、Central china、South china、 East china、 Northwest and Southwest.

2) The factors which may affect the travelers to choose the travel route

They include: weather condition information, the severity of traffic incident or congestion on the original route, the traffic condition of the alternative route, the delay time on original route, the traffic stops and intersection in the alternative route, the travel time saved by the alternative route, the familiarity with the road network.

Taking into account the characteristics of the characteristics of the driver groups, the professional drivers and the travelers often taking taxi and Public car as the main trip mode are chosen for investigation objects.

In order to avoid the shortcomings of the roadside field investigation method, which are caused by the investigation objects' hateful fill, and the recovery rate of the mail questionnaire investigation method is very low. From January to February 2013, the uncertain time investigation was selected, which was implemented by traffic engineering undergraduates. The scope of the investigation is the whole country. Of the 1800 questionnaires which were handed out, 1576 were returned, 1306 were valid questionnaires, i.e. the response rate was $72.56 \%$.

\section{Preliminary Analysis of Survey Results}

SP survey data has been analyzed through the statistical models. The survey results of the socio-economic characteristics of the travelers are shown in Table 1.

Table 1 Characteristics of samples

\begin{tabular}{llr}
\hline Field & Subfield & Samp \\
\hline Gender & male & 69.98 \\
& female & 30.02 \\
Age & <30(voung) & 45.28 \\
& 31 60(midage) & 49.61 \\
Married & $>60$ (old) & 5.12 \\
& ves & 66.24 \\
Education & no & 33.76 \\
Level & primary and junior middle 15.35 \\
& senior high school(shs) & 29.04 \\
& college education & 45.08 \\
City Type & master or doctor(md) & 10.53 \\
& megalonolis & 8.12 \\
& big citv & 41.85 \\
Regional Type & medium-sized citv & 28.05 \\
& small citv & 21.99 \\
& northeast & 55.22 \\
& north china & 16.14 \\
& central china & 11.12 \\
& south china & 1.38 \\
& east china & 14.67 \\
& northwest & 1.18 \\
Monthly & southwest & 0.30 \\
Household & less than 1500(idsr) & 15.65 \\
Income (Y) & 1501-3000(dsr) & 40.94 \\
Vehicle Type & 3001-8000(gsr) & 35.14 \\
& more than $8000($ igsr) & 8.28 \\
& private car & 17.32 \\
& public car & 23.62 \\
& taxi & 8.46 \\
& truck & 19.69 \\
\hline & &
\end{tabular}




\section{Travel Route Choice Model}

This section presents the modeling framework for analyzing the factors influencing the travel route choice. In this study, travel route choice is defined with respect to the current route. The current route is defined as the actual pre-trip chosen on the previous day for pre-trip switching, and en-route decisions on the given day.

There are a lot of factors that influence the travel route choice. The travelers of different gender, age, education level, household incomes have the different reactions to the same traffic information. Since most of these factors are non-continuity, multivariate analysis based on discrete choice analysis is used for analyzing the factors which may influence the travel route choice. The factors are state-dependence and auto-correlation, which may result in inconsistent estimates and erroneous inferences ${ }^{[22]}$. For this reason, mixed logit model is chosen.

Mixed logit model is a highly flexible model that can approximate any random utility model [12]. It obviates the three limitations of standard logit by allowing for random taste variation, unrestricted substitution patterns, and correlation in unobserved factors over time. Unlike probit model, it is not restricted to normal distributions. Its derivation is straight forward and simulation of its choice probabilities is computationally simple. Like probit model, the mixed logit model has been known for many years but has only become fully applicable since the advent of simulation.

A detailed description of mixed logit is available in Train (2003) and Walker (2001). The specification of a random coefficient mixed logit model uses the following utility specification (for a decision maker $n$ choosing alternative route $j$ from a choice set of $\mathbf{J}$ alternatives):

$$
U_{n j}=\beta_{n} x_{n j}+\sigma_{j} \varepsilon_{n j}+v_{n j} \beta_{n}: f\left(\beta_{n} \mid \theta\right)
$$

Where $U_{n j}$ is the utility of alternative $j$ of decision makes, $n . x_{n j}$ is observed variables that relate to the alternative, $j$, and decision maker, $n$, which are referred to the factors influencing the traveler

route choice. $\beta_{n}$ is a vector of coefficients of these variables. $f\left(\beta_{n} \mid \theta\right)$ is density function of $\beta_{n}$ under the overall parameters $\theta$. $\varepsilon_{n j}$ is a Gaussian, zero-mean error term with a standard deviation $\sigma_{j}$; and $v_{n j}$ is a zero-mean, random term that is iid extreme value. If there is no preference difference, under the condition of $\beta_{n}$ which is fixed, the conditional selection probability of the decision makers is:

$$
P_{n i}\left(\beta_{n}\right)=\frac{\exp \left(\beta_{n} x_{n i}\right)}{\sum_{J} \exp \left(\beta_{n} x_{n j}\right)}
$$

In the mixed logit model, $\beta_{n}$ is a random variation. Thus the conditional selection probability of the decision makers is gained, under the condition of existing random preference differences. The final form of mixed logit model is:

$$
P_{n i}^{\prime}=\int P_{n i} f\left(\beta_{n} \mid \theta\right) d \beta=\int\left(\frac{\exp \left(\beta_{n} x_{n i}\right)}{\sum_{j} \exp \left(\beta_{n} x_{n j}\right)}\right) f\left(\beta_{n} \mid \theta\right) d \beta
$$

The selective probability of Mixed logit model can be seen as the weighted average of the multidimensional logit model selective probability, which is determined by the distribution of the density function $f\left(\beta_{n} \mid \theta\right) . \theta$ is the unknown characteristic parameters as a function of the density, such as the mean and variance of a normal distribution. $\beta_{n}$ is random variable, which can follow a normal distribution, lognormal, $\mathrm{S}_{\mathrm{B}}$ distribution, uniform distribution and triangular distribution. In actual study, the use of normal and $S_{B}$ distribution, due to the normal distribution distributed on both sides at zero. According to the logic and the actual data, $\beta_{n}$ are calibrated by $S_{\mathrm{B}}$ distribution.

\section{Modeling Results}

\section{Overall Object Model}

Because some of the influencing factors have a strong correlation among them, the certain factors have not significantly predicted for the decision makers. So the influencing factors need to 
be filtered. The travelers of total samples are chosen as overall objects, the significant independent variables used step by step logit regression include: travelers' socio-economic characteristics, the severity of the traffic incident or congestion (stic), travel time saved by an alternative route (ttsar, including sttsar (travel time saved by an alternative route [0-15 min]), mttsar (travel time saved by an alternative route [16-30 $\mathrm{min}]$ ) and lttsar (travel time saved by an alternative route [>30 min])), familiarity with the road network (frn) and traffic information evaluation and attitude (trea). The travelers' socio-economic characteristics include: the regional type, the city type, age, gender, full-time job, education level and the vehicle type. After the inspection, the variance inflation factor (VIF) of the independent variables is less than the critical point, so there are not significant multicollinearity among the independent variables. Mixed logit prediction model is built (see in Table 2).

Table 2 the results of mixed logit model in the whole country

\begin{tabular}{cccccc}
\hline The factors & B & Std. & Wald & Sig & Exp(B \\
\hline northeast & 17.771 & 1.068 & 27.0 & 0.00 & 5.220 \\
north china & 16.691 & 1.111 & 22.6 & 0.00 & 1.774 \\
central china & 17.822 & 1.099 & 26.0 & 0.00 & 5.493 \\
south china & 19.420 & 1.222 & 25.7 & 0.00 & 2.715 \\
east china & 17.681 & 1.087 & 26.4 & 0.00 & 4.772 \\
northwest & 17.238 & 1.000 & 24.3 & 0.00 & 3.064 \\
megalopolis & 0.655 & 0.182 & 12.9 & 0.03 & 1.925 \\
big cities & 0.615 & 0.213 & 8.34 & 0.03 & 1.850 \\
medium-size & 0.497 & 0.473 & 1.10 & 0.03 & 1.644 \\
small cities & 0.267 & 0.260 & 1.05 & 0.03 & 1.306 \\
young & -1.696 & 0.293 & 33.5 & 0.00 & 0.183 \\
midage & -1.111 & 0.232 & 23.0 & 0.00 & 0.329 \\
Gender & 0.756 & 0.546 & 1.91 & 0.00 & 2.129 \\
full-time job & 0.316 & 0.178 & 3.16 & 0.03 & 1.371 \\
pims & -0.265 & 0.629 & 5.41 & 0.00 & 0.767 \\
shs & -0.574 & 0.600 & 8.45 & 0.00 & 0.563 \\
ceb & -0.163 & 0.093 & 21.6 & 0.00 & 0.849 \\
md & -0.007 & 0.081 & 13.2 & 0.00 & 0.993 \\
private car & 0.672 & 0.246 & 7.49 & 0.00 & 1.958 \\
Public car & 0.164 & 0.271 & 2.92 & 0.00 & 1.178 \\
taxi & 0.484 & 0.253 & 2.30 & 0.00 & 1.622 \\
stic & 1.334 & 0.251 & 28.3 & 0.00 & 3.796 \\
sttsar & 0.728 & 2.231 & 5.24 & 0.00 & 2.071 \\
mttsar & 0.436 & 1.201 & 7.61 & 0.00 & 1.547 \\
lttsar & 1.218 & 0.468 & 20.2 & 0.00 & 3.380 \\
frn & 0.529 & 0.231 & 5.24 & 0.02 & 1.697 \\
trea & 1.538 & 0.342 & 18.5 & 0.00 & 4.655 \\
Fitting degree & $\mathrm{AIC}=145.2$ & SC $=165.4$ \\
\hline & LR=113.2 & LRI=0.88 \\
\hline
\end{tabular}

From the fitting degree of overall object model, likelihood ratio index (LRI) is 0.88, LRI value closes to 1 that the model fits better. The values of Akaike Information Criterion (AIC) and Schwarz Criterion (SC) are both small. The smaller they are, the better the model fits. The single independent variable influences the selection probability of the alternative route as follows.

1) regional type

The severity of the traffic problems faced by travelers are very different with the level of economic development in the different regions, the concept of time travel and time values are also different. The estimation coefficients are relatively large and positive, which indicates that the the regional type has a significant positive correlation with the selection probability of the travel route choice. There are not the results of this research at home and abroad. 


\section{2) city type}

The travelers in the different cities face the different severity of the traffic problems, which leads to the different degree of the travelers' concern about the alternative route. The regression coefficients show that the travelers in the large cities are more willing to choose the alternative route, e.g., the city type is very important to choose the alternative route. At present, there are not the results of this research at home and abroad.

3) age

It has a negative correlation with the selection probability of the alternative route, which suggests that with the age increasing, the probability of the alternative route which are travelers selected according to the traffic information will decline, e.g., compare with the old travelers, the young travelers are more willing to follow traffic information, and change the travel route. This conclusion is consistent with the domestic and foreign researchers ${ }^{[24 \sim 25]}$.

4) gender

Data shows that male travelers are more willing to change the travel route than female travelers. The reason is that male travelers have less patience, more sensitive for the delay, and more likely to choose an alternative route. The result is consistent with the foreign researchers' conclusion ${ }^{[24,26]}$.

5) full-time job

If the travelers' work is the full-time job, the travelers are more willing to choose the alternative route. The likely explanation for this phenomenon is: compared to the travelers of the non-full-time job, the travel time value of travelers whose work are the full-time job is higher, and more sensitive for the delay, so the travelers of the full-time work are more willing to follow the recommendations of the alternative route. The fitting parameters indicate that the selection probability of the full-time work travelers' alternative route is as 1.371 time as the selection probability of the non-full-time work travelers' alternative route.

6) education level

The regression coefficient is negative, which shows that with the travelers' education level increasing, the selection probability of the alternative route will reduce. The fitting parameters indicate that, the selection probability of the travelers whose education level is the primary, middle and high school is significantly higher than the high degree travelers' selection probability. This is similar to previous research results at home and abroad ${ }^{[23]}$.

7) vehicle type

Data shows that, the travelers who often drive or take the taxi as prominent travel mode have a high selection probability of the alternative route, but travelers who drive public cars and trucks have a low probability. The phenomenon has a great relationship with the drivers' characteristics.

8) the severity of the traffic incident or congestion

Different travelers tolerate the different severity of the traffic incident or congestion. If the travelers can bear the greater severity of the incident or congestion, they have a low selection probability of the alternative route. That is, the severity of traffic incident or congestion has a positive correlation with the probability choosing the alternative route. The fitting parameters show that, when the severity degree of the traffic incident or congestion increases one level, the selection probability of the alternative route will be increased by 3.796 times.

9) travel time saved by the alternative route

It has a positive correlation with the selection probability of the alternative route. It shows that, if the alternative route can save longer travel time, the travelers have a higher selection probability of the alternative route. That is, travel time saved by the alternative route has a positive role in promoting the choice probability of the alternative route. It with the foreign research results is consistent $^{[25]}$.

10) familiarity with the road network

It and the selection probability of the alternative route are a positive correlation, e.g., with the road network familiarity increasing, the probability of the alternative route also increases. This is consistent with the results of Haitham and Conquest. The familiarity which the road network 
increases an additional level, the selection probability will be increased by 1.697 times.

11) traffic information evaluation and attitude

The regression coefficient shows that traffic information evaluation and attitude have a significant relationship with the selection probability of the alternative route. With the improvement of the traffic information accuracy, the travelers more trust traffic information, accept the available information and change the travel route according to the information content. This research result is consistent with the results at home and abroad ${ }^{[23]}$.

\section{Typical Object Model}

\section{Typical Region Object Model}

According to the regional characteristics, the survey area can be divided into the northeast, north china, and so on, total seven large regions. When mixed logit model is established, the sample size is less than 100, the estimation risk is bigger (Wang et al. 2001). Form table 1, it is known that the travelers in the northeast and east area choose as the analysis objects, the typical regional travel route choice probability model is established.

1)Northeast travel route choice model

The travelers in northeast region are chosen as the analysis object, the significant variables are gained by the use of a stepwise logit regression method. Where the significant variables include: the travelers' socio-economic characteristics, travel time saved by the alternative route (ttsar), the delay time on the original route (dtor), the severity of the traffic incident or congestion (stic), the traffic stops or intersection in the alternative route (tsiar), and familiarity with the road network (frn). The travelers' socio-economic characteristics include: age, gender, the vehicle type. After inspection, the variance inflation factor (VIF) of independent variables is less than the critical point, so there is not significant multicolorlinearity among the independent variables. The estimation results of northeast object model are shown in Table 4.

From the fitting degree of the model, likelihood ratio index LRI is 0.82 , LRI value closes to 1 that the model fits better. Akaike Information Criterion (AIC) and Schwarz Criterion (SC) are both small, the smaller they are, the better the model fits (Wang et al. 2001). The effect of a single independent variable on the alternative route selection probability is similar to overall object model. Therefore, we no longer explain detailed.

Table 4 the results of mixed logit model in northeast region

\begin{tabular}{cccccc}
\hline The factors & B & $\begin{array}{c}\text { Std. } \\
\text { Error }\end{array}$ & Wald & Sig & $\begin{array}{c}\operatorname{Exp}(\mathrm{B} \\
)\end{array}$ \\
\hline young & -0.71 & 0.392 & 7.313 & 0.00 & 0.490 \\
midage & -0.41 & 0.154 & 3.215 & 0.00 & 0.662 \\
gender & 0.513 & 0.142 & 4.233 & 0.00 & 1.670 \\
private car & 0.396 & 0.682 & .337 & 0.03 & 1.485 \\
Public car & 0.276 & 0.731 & .424 & 0.01 & 1.317 \\
taxi & 0.471 & 0.713 & .437 & 0.03 & 1.602 \\
sttsar & 0.132 & 1.613 & .007 & 0.03 & 1.141 \\
mttsar & 0.814 & 1.131 & .518 & 0.04 & 2.256 \\
lttsar & 1.218 & 0.468 & 20.247 & 0.03 & 3.380 \\
dtor & 2.064 & 0.994 & 4.316 & 0.03 & 7.877 \\
stic & 17.75 & 0.653 & 739.53 & 0.00 & 5.119 \\
tsiar & 0.463 & 0.643 & .520 & 0.04 & 1.590 \\
frn & 0.460 & 0.245 & 3.508 & 0.00 & 1.584 \\
Fitting degree & AIC $=118.2$, & SC $=134.4$, \\
LR=198.3. & LRI $=0.82$ \\
\hline
\end{tabular}

2) East china travel route choice model

The travelers in east region are chosen as the analysis objects, the significant variables are 
gained by the use of a stepwise logit regression method. The significant variables include: the travelers' socio-economic characteristics, travel time saved by an alternative route (ttsar), the weather conditions (wc), familiarity with the road network (frn), and the length and road type of the alternative route (ltar). The travelers' socio-economic characteristics include: education level and the vehicle type. After the inspection, the variance inflation factor (VIF) of independent variable is less than the critical point, so there is not significant multicollinearity among the independent variables. The estimation results of east china object model are shown in Table 5. From the value of likelihood ratio index LRI, Akaike Information Criterion (AIC) and Schwarz Criterion (SC), they show that the fitting degree of the model is better.

Table 5 the estimation results of mixed logit model in east region

\begin{tabular}{|c|c|c|c|c|c|}
\hline The factors & B & $\begin{array}{l}\text { Std. } \\
\text { Error }\end{array}$ & Wald & Sig & $\operatorname{Exp}(B)$ \\
\hline pjms & -1.351 & 2.462 & 0.337 & 0.005 & 0.259 \\
\hline shs & -0.373 & 0.934 & 0.159 & 0.007 & 0.688 \\
\hline ceb & -0.219 & 0.854 & 0.066 & 0.024 & 0.803 \\
\hline private car & 0.396 & 1.005 & 0.291 & 0.046 & 1.486 \\
\hline Public car & 0.276 & 0.867 & 1.264 & 0.015 & 1.318 \\
\hline $\operatorname{taxi}$ & 0.896 & 1.206 & 1.597 & 0.038 & 2.449 \\
\hline sttsar & 1.218 & 0.468 & 20.247 & 0.000 & 3.380 \\
\hline mttsar & 1.881 & 0.705 & 7.416 & 0.006 & 6.560 \\
\hline lttsar & 1.921 & 0.620 & 9.212 & 0.002 & 6.828 \\
\hline wc & 1.344 & 1.158 & 1.347 & 0.006 & 3.834 \\
\hline frn & 0.463 & 0.643 & 0.520 & 0.004 & 1.589 \\
\hline ltar & 5.490 & 2.450 & 5.023 & 0.005 & 242.257 \\
\hline Fitting degree & & \multicolumn{2}{|c|}{$\begin{array}{c}\mathrm{AIC}=153.87 \\
\mathrm{LR}=108.3\end{array}$} & \multicolumn{2}{|c|}{$\begin{array}{c}\mathrm{SC}=159.88 \\
\mathrm{LRI}=0.81\end{array}$} \\
\hline
\end{tabular}

From the table 3 , table 4 and table 5, travel route choice probability model whose analysis objects are travelers in the northeast and the east China area have great difference with the overall object model. Compared with overall object model, the typical object model has a certain differences in independent variables and estimation coefficient. In addition, it shows that the influence factors of the travel route choice are more comprehensive, which are estimated by overall object model. This suggests that the regional characteristic is one of the important factors which impact the travelers to change the travel route. Compared with table 4 and 5, we know that there are certain differences in estimation coefficient and independent variables between two typical object models, which are established based on the regional characteristic. This shows that, the travelers in different regions consider that the main factors influencing the travel route are not identical, there are some differences. There are the same factors influencing the travel route choice in difference regions, but its estimation coefficient is different. This indicates that the same travel route choice factors have different effects in different regions.

\section{Typical City Object Model}

According to the city type, the survey area can be divided into megalopolis, big city, and so on, total four types. Because the sample size of mix logit model was required, the travelers in big city and medium-size city are chosen. The travel route choice probability model of typical city type is established.

(1) Big city travel route choice model

The travelers in big city are chosen as analysis objects, the significant variables are gained by the use of a stepwise logit regression method. The significant variables include: the travelers' 
socio-economic characteristics, the severity of the traffic incident or congestion (stic), the travel time saved by an alternative route (ttsar), the length and road type of the alternative route (ltar), the traffic stops or intersection in the alternative route (tsiar), and familiarity with the road network (frn). The travelers' socio-economic characteristics include: education level and household monthly income. After inspection, the variance inflation factor (VIF) of independent variable is less than the critical point, so there is not significant multicollinearity among the independent variables. The estimation results of the model are shown in Table 6.

Table 6 the estimation results of mixed logit model in big city

\begin{tabular}{cccccc}
\hline The factors & B & Std.Err & Wald & Sig & $\operatorname{Exp}(\mathrm{B}$ \\
\hline pims & -0.957 & 1.147 & 0.69 & 0.00 & 0.384 \\
shs & -0.247 & 0.760 & 0.10 & 0.00 & 0.781 \\
ceb & -0.593 & 0.527 & 1.26 & 0.00 & 0.553 \\
md & -0.396 & 0.682 & 0.33 & 0.01 & 0.673 \\
jdsr & 0.940 & 1.914 & 0.24 & 0.02 & 2.559 \\
dsr & 1.406 & 1.614 & 0.75 & 0.03 & 4.080 \\
gsr & 0.763 & 1.459 & 0.27 & 0.01 & 2.145 \\
stic & 32.531 & 2.078 & 245. & 0.00 & 1.343 \\
sttsar & 0.132 & 1.613 & 0.00 & 0.00 & 1.141 \\
mttsar & 0.814 & 1.131 & 0.51 & 0.00 & 2.256 \\
lttsar & 1.218 & 0.468 & 20.2 & 0.00 & 3.380 \\
ltar & -3.489 & 1.826 & 3.65 & 0.00 & .031 \\
tsiar & -10.14 & 34.877 & 0.08 & 0.00 & 3.948 \\
frn & 0.399 & 1.881 & 0.04 & 0.00 & 1.490 \\
Fitting degree & AIC $=163.2$ & SC $=170.4$ \\
& LR=194.3 & LRI $=0.84$ \\
\hline
\end{tabular}

From the fitting degree of the model, likelihood ratio index LRI is 0.84 , LRI value closes to 1 that the model fits better. Akaike Information Criterion (AIC) and Schwarz Criterion (SC) are both small, they are smaller which the model fits better (Wang et al. 2001). The effect of the single independent variable on the alternative route selection probability is similar to overall object model. Therefore, we no longer explain detailed.

(2) Medium-size city travel choice model

The travelers in medium-size city are selected as analysis objects, the significant variables are gained by the use of a stepwise logit regression method. Where the significant variables include: the travelers' socio-economic characteristics, the delay time on the original route (dtor), traffic incident location and influence scope (tilis), the traffic condition of the alternative route (tcar) and familiarity with the road network ( $f r n)$. The traveler socio-economic characteristics include: occupation, gender and the vehicle type. After inspection, the variance inflation factor (VIF) of independent variable is less than the critical point, so there is not significant multicollinearity among the independent variables. Mixed logit prediction model is built (see in Table 7). From the vaule of likelihood ratio index LRI, Akaike Information Criterion (AIC) and Schwarz Criterion (SC), it shows that the fitting degree of the model is better. 
Table 7 the results of mixed logit model in medium-size city

\begin{tabular}{|c|c|c|c|c|c|}
\hline The factors & B & $\begin{array}{c}\text { Std.Err } \\
\text { or }\end{array}$ & Wald & Sig & $\operatorname{Exp}(B)$ \\
\hline full-time job & 0.786 & 0.383 & 4.206 & 0.001 & 2.194 \\
\hline gender & 0.513 & 0.142 & 4.233 & 0.004 & 1.670 \\
\hline private car & 0.672 & 0.246 & 7.490 & 0.006 & 1.958 \\
\hline public car & 0.364 & 0.271 & 2.923 & 0.007 & 1.439 \\
\hline $\operatorname{tax} \mathrm{i}$ & 0.484 & 0.253 & 2.309 & 0.009 & 1.622 \\
\hline dtor & 2.064 & 0.994 & 4.316 & 0.038 & 7.877 \\
\hline tilis & $\begin{array}{c}17.75 \\
1\end{array}$ & 0.653 & $\begin{array}{c}739.5 \\
39\end{array}$ & 0.000 & $5.119 \mathrm{E} 7$ \\
\hline tcar & 0.483 & 0.643 & 0.520 & 0.004 & 1.621 \\
\hline frn & 0.460 & 0.245 & 3.508 & 0.001 & 1.584 \\
\hline Fitting degree & & $\begin{array}{l}\mathrm{IC}=175 \\
\mathrm{R}=198.3\end{array}$ & & $\begin{array}{l}\mathrm{SC}=136 \\
\mathrm{LRI}=0\end{array}$ & \\
\hline
\end{tabular}

From the table 4, table 6 and table 7 , travel route choice probability model whose analysis objects are travelers in big city and medium-size city have great difference with the overall object model. This suggests that the city type is one of the important factors influencing the travel route choice. The travelers in different type cities consider that the main factors changing travel route are not the same. Comparison with table 6 and 7, we know that there are certain differences in estimation coefficient and independent variables between two typical object models which are established based on the city type. This shows that, the travelers in different type town consider that the main factors of changing travel route are not identical, there are some differences. There are the same factors that influence the travel route choice in difference type cities, but its estimation coefficient is different, this indicates that in different type cities the same travel route choice factors have different effects.

\section{Conclusion}

In this paper, the research has understood how traffic information influences travelers' route choice behavior. The travel behavior data was obtained by Stated Preference Survey. In the survey, the investigation objects are the travelers across the country, each individual was asked for the following information: ( i ) traveler socio-economic characteristics, (ii) the factors which may affect the travelers to choose the alternative route, as well as how the obtained information influenced their travel route choice.

Two models have been estimated: overall object model and typical object model which are based on mixed logit model, and used for analyzing the factors influencing the travel route choice. The models are then verified using survey data from different range. The results confirm that the travelers' social-economic characteristics, the severity of traffic incident, the saving time of alternative route, the delay time on the original route, road network knowledge, and travelers' evaluation and attitude on traffic information are the main factors that impact the route choice behavior. There are some different influencing factors between overall object model and typical object model.

In the presented research, some scholars have used of mixed logit model to study the effect of the traffic information on travel behavior, but in china, there is no scholars who has used of mixed logit model to analyze the factors influencing travel route choice. Based on the advantages of mixed logit model, we used of it to estimate the main factors influencing travel route choice. The results show that the main factors influencing travel route choice in china environment are not consistent with the foreign research results. This indicates that the regional characteristic is one of the important factors which affect travelers to change the travel route. This deduction has also been 
proved in the follow-up study in this paper.

In this paper, we have established typical regional object model and typical city object model which are based on the different survey data. The results of these two class models confirm that the regional characteristic and the city type are very important factors influencing the travel route choice. In the past research, there is not scholar who studies this problem.

The researcher results can provide the basis for traffic information release methods and strategies, and provide the theoretical basis for Advanced Transportation Information Service system (ATIS) evaluation. This article is based on a nationwide sample survey, and the regional characteristics and the city type are the important factors which affect travelers to change the travel route. The application in a special area maybe will be different. Therefore, the practical application should be improved by traffic simulation and repeat experiments, and then a more reasonable model was obtained by inspection correction in the real environment.

\section{Acknowledgements}

The research described in this paper is largely supported by the National Natural Science Foundation of China (Grant No. 71501061). The authors would like to thank anonymous referees for their helpful comments and valuable suggestions which improved the content and composition substantially.

\section{Reference}

[1].Heathington, K.W., Worrall, R.,Hoff, G. Attitudes and behavior of drivers regarding route diversion[R]. 1971,

[2].Ratcliffe, E., A comparison of drivers route choice criteria and those used in current assignment processes, 1970, University of Newcastle upon Tyne.

[3].Huchingson, R., McNees, R.,Dudek, C. Survey of Motorist Route-Selection Criteria (Abridgment)[J]. Transportation Research Record, 1977, 643):

[4].Penersen, D.M. Factors in route selection[J]. Perceptual and motor skills, 1998, 86(3):999-1006.

[5].Duffell, J.,Kalombaris, A. Empirical studies of car driver route choice in Herfordshire[J]. Traffic Engineering and Control, 1988, 29(7/8):

[6].Emmerink, R.H., Nijkamp, P., Rietveld, P.,Van Ommeren, J.N. Variable message signs and radio traffic information: An integrated empirical analysis of drivers' route choice behaviour[J]. Transportation Research Part A: Policy and Practice, 1996, 30(2):135-153.

[7].Abdel-Aty, M.A., Kitamura, R.,Jovanis, P.P. Using stated preference data for studying the effect of advanced traffic information on drivers' route choice[J]. Transportation Research Part C: Emerging Technologies, 1997, 5(1):39-50.

[8].Peeta, S., Ramos, J.L.,Pasupathy, R. Content of variable message signs and on-line driver behavior[J]. Transportation Research Record: Journal of the Transportation Research Board, 2000, 1725(1):102-108.

[9]. Chatterjee, K., Hounsell, N., Firmin, P.,Bonsall, P. Driver response to variable message sign information in London[J]. Transportation Research Part C: Emerging Technologies, 2002, 10(2):149-169.

[10]. Limtanakool, N., Dijst, M.,Schwanen, T. The influence of socioeconomic characteristics, land use and travel time considerations on mode choice for medium-and longer-distance trips[J]. Journal of Transport Geography, 2006, 14(5):327-341.

[11]. Tsirimpa, A., Polydoropoulou, A.,Antoniou, C. Development of a mixed multi-nomial logit model to capture the impact of information systems on travelers' switching behavior[J]. 
Journal of Intelligent Transportation Systems, 2007, 11(2):79-89.

[12]. McFadden, D.,Train, K. Mixed MNL models for discrete response[J]. Journal of applied Econometrics, 2000, 15(5):447-470.

[13]. Bonsall, P.,Parry, T. Using an interactive route-choice simulator to investigate drivers'compliance with route guidance advice [M]. 1991.

[14]. Khattak, A.J., Schofer, J.L.,Koppelman, F.S. Factors influencing commuters'en route diversion behavior in response to delay (with discussion)[J]. Transportation Research Record, 1991, 1318):

[15]. Srinivasan, K.K.,Mahmassani, H.S. Role of congestion and information in trip-makers' dynamic decision processes: Experimental investigation[J]. Transportation Research Record: Journal of the Transportation Research Board, 1999, 1676(1):44-52.

[16]. Khattak, A.J., Koppelman, F.S.,Schofer, J.L. Stated preferences for investigating commuters' diversion propensity[J]. Transportation, 1993, 20(2):107-127.

[17]. Larsen, J.,El-Geneidy, A. A travel behavior analysis of urban cycling facilities in Montréal, Canada[J]. Transportation research part D: transport and environment, 2011, 16(2):172-177.

[18]. Eluru, N., Chakour, V.,El-Geneidy, A.M. Travel mode choice and transit route choice behavior in Montreal: insights from McGill University members commute patterns[J]. Public Transport, 2012, 4(2):129-149.

[19]. Wardman, M. A comparison of revealed preference and stated preference models of travel behaviour[J]. Journal of Transport Economics and Policy, 1988, 71-91.

[20]. Wardman, M., Bonsall, P.,Shires, J. Driver response to variable message signs: a stated preference investigation[J]. Transportation Research Part C: Emerging Technologies, 1997, 5(6):389-405.

[21]. Hensher, D.A. Measurement of the valuation of travel time savings[J]. Journal of Transport Economics and Policy (JTEP), 2001, 35(1):71-98.

[22]. Abdel-Aty, M.,Abdalla, M.F. Modeling drivers' diversion from normal routes under ATIS using generalized estimating equations and binomial probit link function[J]. Transportation, 2004, 31(3):327-348.

[23]. Dia, H.,Panwai, S. Modelling drivers' compliance and route choice behaviour in response to travel information[J]. Nonlinear Dynamics, 2007, 49(4):493-509.

[24]. Al-Deek, H.M., Khattak, A.J.,Thananjeyan, P. A combined traveler behavior and system performance model with advanced traveler information systems[J]. Transportation Research Part A: Policy and Practice, 1998, 32(7):479-493.

[25]. Roider, O., Rudloff, C.,Ray, M. An Individualized Travel Information System for Optimizing Mode and Route Choice behavior of Commuters[J]. Procedia-Social and Behavioral Sciences, 2012, 48(1948-1957.

[26]. Ben-Elia, E.,Shiftan, Y. Which road do I take? A learning-based model of route-choice behavior with real-time information[J]. Transportation Research Part A: Policy and Practice, 2010, 44(4):249-264. 